Robin Nijveldt

Tjeerd Germans

Gerald P. McCann

Aernout M. Beek

Albert C. van Rossum

\section{Semi-quantitative assessment of right ventricular function in comparison to a 3D volumetric approach: A cardiovascular magnetic resonance study}

Received: 19 December 2007

Revised: 6 April 2008

Accepted: 10 April 2008

Published online: 4 June 2008

(C) The Author(s) 2008

R. Nijveldt $(\bowtie) \cdot$ T. Germans ·

A. M. Beek - A. C. van Rossum

Department of Cardiology,

VU University Medical Center,

De Boelelaan 1117,

1081 HV Amsterdam, The Netherlands e-mail: r.nijveldt@vumc.nl

Tel.: +31-20-4442244

Fax: +31-20-4443395

R. Nijveldt · T. Germans ·

A. C. van Rossum

Interuniversity Cardiology Institute of

the Netherlands,

Utrecht, The Netherlands

G. P. McCann

Department of Cardiology,

University Hospitals Leicester,

Leicester, UK
Abstract Right ventricular (RV) volume measurements with cardiovascular magnetic resonance (CMR) is considered the gold standard, but acquisition and analysis remain timeconsuming. The aim of our study was therefore to investigate the accuracy and performance of a semi-quantitative assessment of RV function in CMR, compared to the standard quantitative approach. Seventy-five subjects with pulmonary hypertension (15), anterior myocardial infarction (15), inferior myocardial infarction (15), Brugada syndrome (15) and normal subjects (15) underwent cine CMR. RV end-systolic and enddiastolic volumes were determined to calculate RV ejection fraction (EF). Four-chamber cine images were used to measure tricuspid annular plane systolic excursion (TAPSE). RV fractional shortening (RVFS) was calcu- lated by dividing TAPSE by the RV end-diastolic length. RV EF correlated significantly with TAPSE $(\mathrm{r}=0.62$, $\mathrm{p}<0.01)$ and RVFS $(r=0.67, \mathrm{p}<0.01)$. Sensitivity to predict RV dysfunction was comparable between TAPSE and RVFS, with higher specificity for RVFS, but comparable areas under the ROC curve. Intra- and inter-observer variability of RV EF was better than TAPSE $(3 \% / 4 \%$ versus $7 \% / 15 \%$, respectively). For routine screening in clinical practice, TAPSE and RVFS seem reliable and easy methods to identify patients with RV dysfunction. The 3D volumetric approach is preferred to assess RV function for research purposes or to evaluate treatment response.

Keywords Cardiovascular magnetic resonance $\cdot$ Right ventricular function - TAPSE

\section{Introduction}

Right ventricular (RV) function has proven to be of significant importance in a wide range of cardiac disease. It is a major determinant of clinical outcome in chronic heart failure, a prognostic marker for adverse outcome after acute myocardial infarction and an important measure in the evaluation and follow-up of patients with pulmonary arterial hypertension [1-4]. Thus, the need for diagnosis of RV dysfunction is evident. Various (non)invasive imaging modalities are available for the evaluation of RV function [5-10], of which cardiovascular magnetic resonance (CMR) is considered the gold standard for quantitative assessment $[11,12]$. Although assessment of RV volumes and function using CMR is highly accurate and reproducible [13, 14], both acquisition and analysis are still time-consuming and require dedicated post-processing software. In clinical practice, 2D echocardiography is generally used to acquire functional information of the RV. A quick semi-quantitative approach to assess RV function in 2D echocardiography is to measure the tricuspid annular plane systolic excursion (TAPSE), which can easily be obtained, and correlates well with RV function on radionuclide angiography [15-17].

The increasing interest and availability of clinical CMR generates the need for fast and accurate methods to obtain information on RV function and dimensions. For left ventricular analysis, geometrical models have been developed to accurately determine left ventricular function and 
dimensions and to substantially reduce acquisition and analysis duration [18]. However, this approach often fails in RV analysis, while its geometrical complexity impedes the accuracy of such models to determine function and volumes. Some clinicians therefore measure a modified TAPSE, adapted from echocardiography. However, it is unknown whether this semi-quantitative approach can be legitimately used in CMR and how it relates to the quantitative assessment of RV function. Furthermore, it is disputable whether an absolute TAPSE value accurately represents RV function in all patients, while this measure disregards the dimensions of the RV. Therefore, it may be more accurate to use a semi-quantitative measure that involves RV dimensions to determine RV function.

The present study was designed to determine the accuracy of a semi-quantitative assessment of RV function in CMR and to evaluate its diagnostic performance for identifying different degrees of RV dysfunction. Furthermore, we sought to investigate the intra-observer and interobserver reproducibility of this method, compared to the standard quantitative approach.

\section{Materials and methods}

\section{Subject selection}

Patients for the study were recruited from the clinic at the cardiology department of the VU University Medical Center, Amsterdam, the Netherlands. We prospectively investigated 60 patients ( 39 men; mean age, $50 \pm 13$ years), consecutively enrolled per each patient group. The study population consisted of 15 patients with pulmonary arterial hypertension, 15 patients with anterior acute myocardial infarction (AMI), 15 patients with inferior AMI and 15 patients with Brugada syndrome. Pulmonary arterial hypertension (11 idiopathic, 4 systemic sclerosis) was confirmed by cardiac catheterization (mean pulmonary artery pressure, $54 \pm$ $16 \mathrm{mmHg}$ ). Patients with AMI were scanned $5 \pm 2$ days after primary percutaneous coronary intervention with stenting and were treated with aspirin, heparin, abciximab, clopidogrel, statins, beta-blockade and ACE-inhibitors. The Brugada syndrome was confirmed by typical ECG-pattern and a positive ajmaline test [19]. We also included 15 healthy controls without cardiac history or risk factors in the study (5 men; mean age, $41 \pm 12$ years). Exclusion criteria comprised contraindications for CMR (e.g., claustrophobia, pacemakers, very irregular heart rhythm and intracerebral aneurysm clips). The study was approved by the local ethics committee, and all patients gave written informed consent.

\section{CMR study protocol}

CMR examination was performed on a 1.5-T clinical MR system (Magnetom Sonata, Siemens, Erlangen, Germany) using a four-element phased-array cardiac receiver coil. ECG-gated cine imaging was performed using a balanced steady-state free precession pulse sequence, during repeated breath-holds of approximately $10 \mathrm{~s}$. Long axis slices were acquired in the three standard views (four-, three- and two-chamber view), as well as a stack of short axis slices with a typical slice thickness of $5 \mathrm{~mm}$ and a gap of $5 \mathrm{~mm}$ between the slices, fully covering both ventricles from base to apex. MR parameters were as follows: temporal resolution between 35 to $50 \mathrm{~ms}$, typical voxel size $1.5 \times$ $1.8 \times 5.0 \mathrm{~mm}^{3}$, excitation angle $60^{\circ}$, receiver bandwidth $930 \mathrm{~Hz} /$ pixel, TR/TE 3.2/1.6 ms, matrix $256 \times 156$.

\section{Data analysis and definitions}

CMR data for the assessment of RV volumes were analyzed on a personal computer using dedicated software (Mass, Medis, Leiden, the Netherlands). On all short axis cine slices, the endocardial borders of the RV were outlined manually on end-diastolic (first cine phase of the R-wave triggered acquisition) and end-systolic (image phase with smallest cavity area) images, excluding trabeculae and papillary muscles. RV end-diastolic volumes (EDV, ml) and RV end-systolic volumes (ESV, $\mathrm{ml}$ ) were calculated by summation of discs, from which RV ejection fraction (EF, $\%$ ) could be derived. TAPSE measurements were calculated on a separate workstation (Centricity Radiology v6.1, GE Medical Systems, Zeist, the Netherlands). On the fourchamber view, the distance between the cutting edge of the tricuspid annulus with the RV free wall and the RV apex was measured in end-diastole (end-diastolic length, EDL, $\mathrm{mm}$ ) and end-systole (ESL, mm) (Fig. 1). TAPSE was defined as the difference between RV EDL and ESL. An additional relative measure was obtained, which corrects TAPSE for the length of the RV. This right ventricular fractional shortening (RVFS) was calculated as follows:

$\operatorname{RVFS}(\%)=\frac{(E D L-E S L)}{E D L} * 100$

To test intra-observer and inter-observer variability of RV EF and TAPSE measurements, analyses were repeated by the same investigator, and a second investigator respectively on the same images in 25 subjects, from 5 consecutive subjects in each group (RN, TG). Analysis of the scans was performed in random order, with the investigators blinded to medical history of the subject and previous results.

\section{Statistical analysis}

Continuous variables with normal distribution are expressed as mean $\pm \mathrm{SD}$. Analysis of variances (ANOVA) was used to compare differences between patient groups, and 


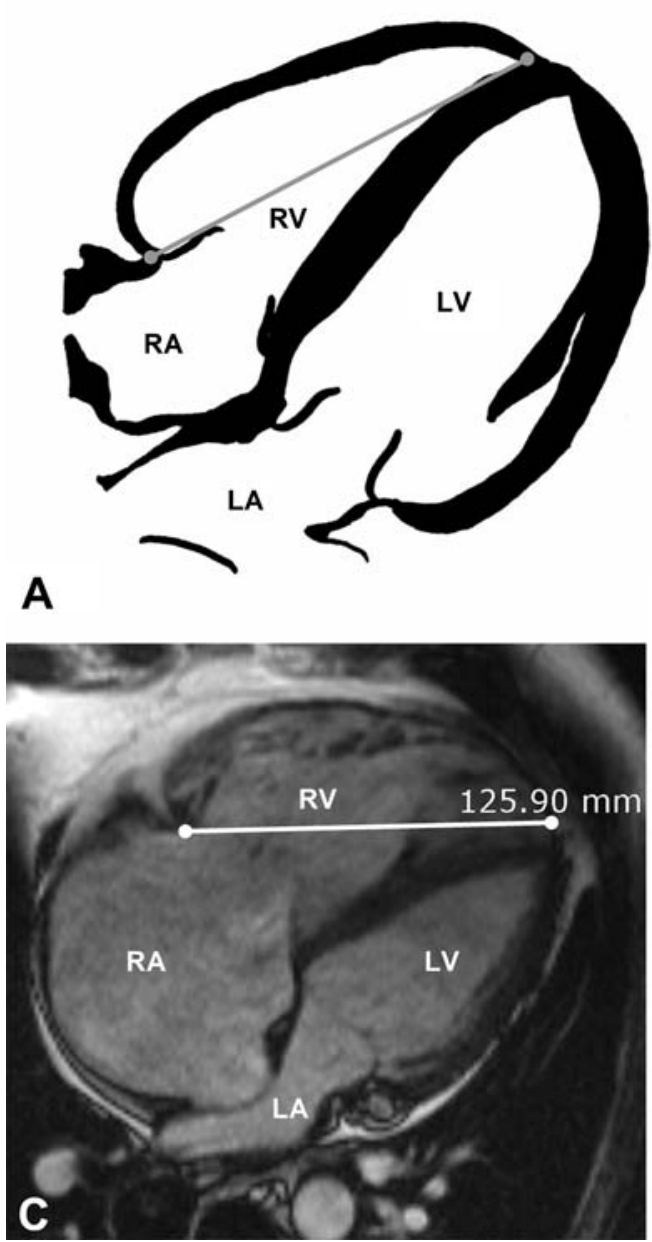

Fig. 1 Schematic figure of TAPSE measurement using a fourchamber cine image in end-diastole (A) and end-systole (B). TAPSE is calculated by subtracting the right ventricular end-systolic length (grey line in panel B) from the end-diastolic length (gray line in panel A). Clinical example of a four-chamber cine image in end-

Tukey's post-hoc test was used for pair-wise comparison of means. Pearson's correlation coefficients (r) were calculated for the relation between RV EF, TAPSE and RVFS. Correlation coefficients were compared using a Fisher's Z transformation. The TAPSE and RVFS value with the highest sensitivity and specificity for predicting RV dysfunction were calculated for RV EF $<35 \%,<40 \%$ and $<45 \%$, using receiver-operating characteristic (ROC) analysis. Comparison between ROC curves following the method described by Hanley and McNeil [20] was performed using Analyse-it Clinical Laboratory 1.73 (Analyse-It Software, Ltd.).

Intra-observer and inter-observer variability of RV EF and TAPSE measurements was assessed as previously described by Bland and Altman [21], and the coefficients of variability (SD of the differences of two measurements divided by their mean) were calculated. A one-sample $t$ test

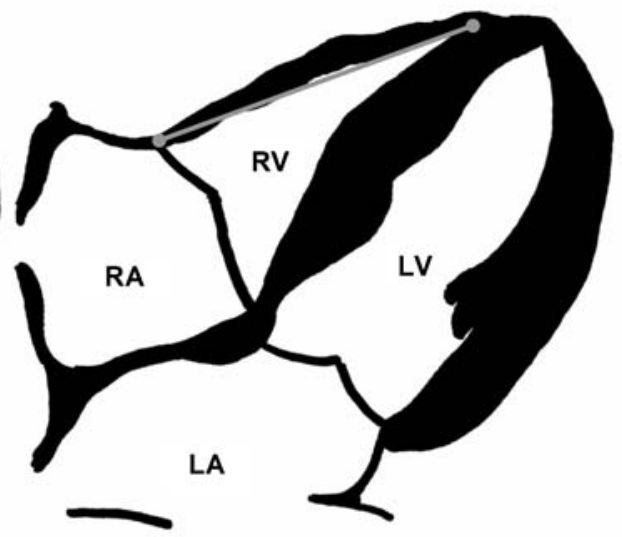

B

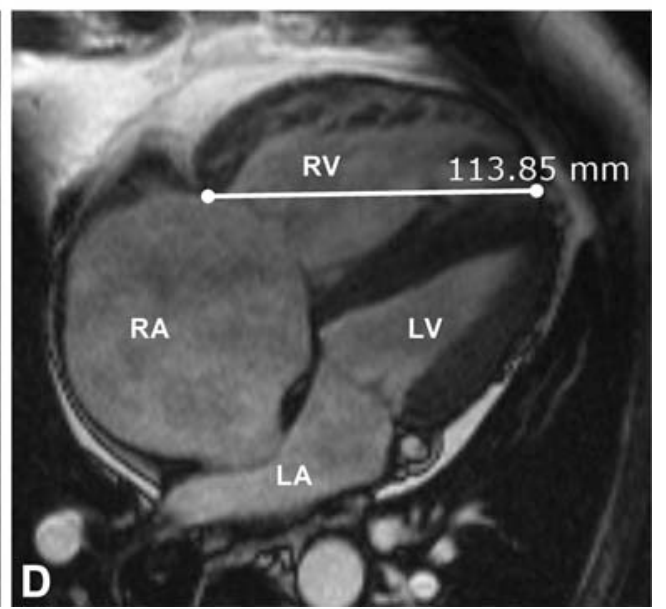

diastole (C) and end-systole (D) in a patient with pulmonary hypertension. The TAPSE measures $125.90-113.85=12.05 \mathrm{~mm}$, and the RVFS is $\frac{(125.90-113.85)}{125.90} * 100=9.57 \%$. RA: right atrium, $\mathrm{RV}$ : right ventricle, LA: left atrium, LV: left ventricle

against zero was performed to test for statistical significance of the observed differences in intra-observer and inter-observer variability.

All statistical tests were two-sided with a significance level of $\mathrm{p}<0.05$. SPSS 12.0.1 for Windows (SPSS Inc.) was used for all analyses, except for the comparison between ROC curves.

\section{Results}

RV volumes, EF, lengths, TAPSE and RVFS are listed in Table 1 according to patient groups. Healthy volunteers and patients with Brugada syndrome were significantly younger than patients with anterior/inferior AMI $(\mathrm{p}<$ 0.05 ). Patients with anterior AMI had larger infarcts than patients with inferior AMI $(21 \pm 12$ versus $13 \pm 9$ percent of 
Table 1 Subject characteristics and CMR measurements of the right ventricle per patient group and as a whole

\begin{tabular}{|c|c|c|c|c|c|c|}
\hline & Controls $(n=15)$ & PHT $(n=15)$ & Anterior AMI $(n=15)$ & Inferior AMI $(n=15)$ & Brugada $(n=15)$ & Total $(n=75)$ \\
\hline Age (years) & $41 \pm 12$ & $47 \pm 13$ & $56 \pm 10$ & $55 \pm 10$ & $42 \pm 12$ & $48 \pm 13$ \\
\hline Peak CK (U/1) & - & - & $3,755 \pm 1,856$ & $2,736 \pm 1,939$ & - & - \\
\hline Infarct size (\% LV) & - & - & $22 \pm 12$ & $13 \pm 9$ & - & - \\
\hline EDV (ml) & $192 \pm 37$ & $248 \pm 93$ & $158 \pm 34$ & $175 \pm 57$ & $188 \pm 47$ & $192 \pm 64$ \\
\hline $\operatorname{EDVi~}\left(\mathrm{ml} / \mathrm{m}^{2}\right)$ & $103 \pm 15$ & $130 \pm 38$ & $80 \pm 14$ & $87 \pm 27$ & $101 \pm 17$ & $100 \pm 29$ \\
\hline $\mathrm{ESV}(\mathrm{ml})$ & $96 \pm 20$ & $188 \pm 97$ & $79 \pm 24$ & $89 \pm 31$ & $97 \pm 28$ & $110 \pm 62$ \\
\hline $\operatorname{ESV}$ i $\left(\mathrm{ml} / \mathrm{m}^{2}\right)$ & $52 \pm 10$ & $98 \pm 44$ & $40 \pm 10$ & $44 \pm 15$ & $52 \pm 12$ & $57 \pm 30$ \\
\hline $\mathrm{SV}(\mathrm{ml})$ & $96 \pm 21$ & $61 \pm 30$ & $79 \pm 19$ & $86 \pm 30$ & $92 \pm 24$ & $83 \pm 27$ \\
\hline $\mathrm{SV}$ i $\left(\mathrm{ml} / \mathrm{m}^{2}\right)$ & $51 \pm 8$ & $32 \pm 15$ & $40 \pm 10$ & $42 \pm 14$ & $49 \pm 10$ & $43 \pm 13$ \\
\hline $\mathrm{EF}(\%)$ & $50 \pm 5$ & $27 \pm 14$ & $51 \pm 8$ & $49 \pm 8$ & $49 \pm 7$ & $45 \pm 13$ \\
\hline EDL (mm) & $91 \pm 8$ & $103 \pm 12$ & $93 \pm 10$ & $98 \pm 13$ & $95 \pm 9$ & $96 \pm 11$ \\
\hline ESL (mm) & $69 \pm 7$ & $89 \pm 13$ & $76 \pm 12$ & $78 \pm 11$ & $72 \pm 9$ & $77 \pm 12$ \\
\hline TAPSE (mm) & $23 \pm 3$ & $15 \pm 6$ & $18 \pm 5$ & $20 \pm 4$ & $23 \pm 4$ & $20 \pm 5$ \\
\hline RVFS (\%) & $25 \pm 3$ & $14 \pm 7$ & $19 \pm 6$ & $21 \pm 3$ & $24 \pm 4$ & $21 \pm 6$ \\
\hline
\end{tabular}

PHT: pulmonary arterial hypertension, AMI: acute myocardial infarction, CK: creatine kinase, LV: left ventricle, EDV: end-diastolic volume, ESV: end-systolic volume, SV: stroke volume, -i: indexed for body surface area, EF: ejection fraction, EDL: end-diastolic length, ESL: end-systolic length, TAPSE: tricuspid annular plane systolic excursion, RVFS: right ventricular fractional shortening

Values are expressed as mean $\pm \mathrm{SD}$

the left ventricle, $p<0.05$ ). RV EDVi (indexed for body surface area) and RV ESVi were higher and RV EF was lower in patients with pulmonary arterial hypertension compared to patients with anterior/inferior AMI and Brugada syndrome $(\mathrm{p}<0.05)$. RV ESVi, RV EDL and
RV ESL were significantly higher and RV EF, TAPSE and RVFS lower in patients with pulmonary hypertension than in healthy volunteers $(p<0.05)$. There were no differences in $\mathrm{RV}$ indices between patients with anterior or inferior AMI $(\mathrm{p}=\mathrm{ns})$.
A

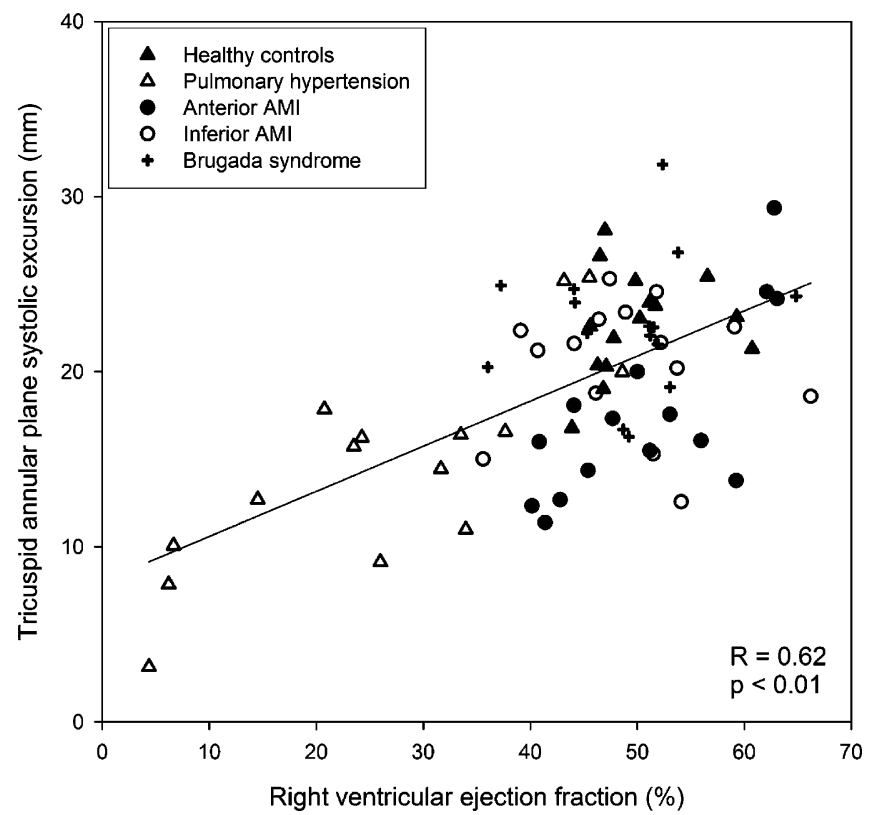

B

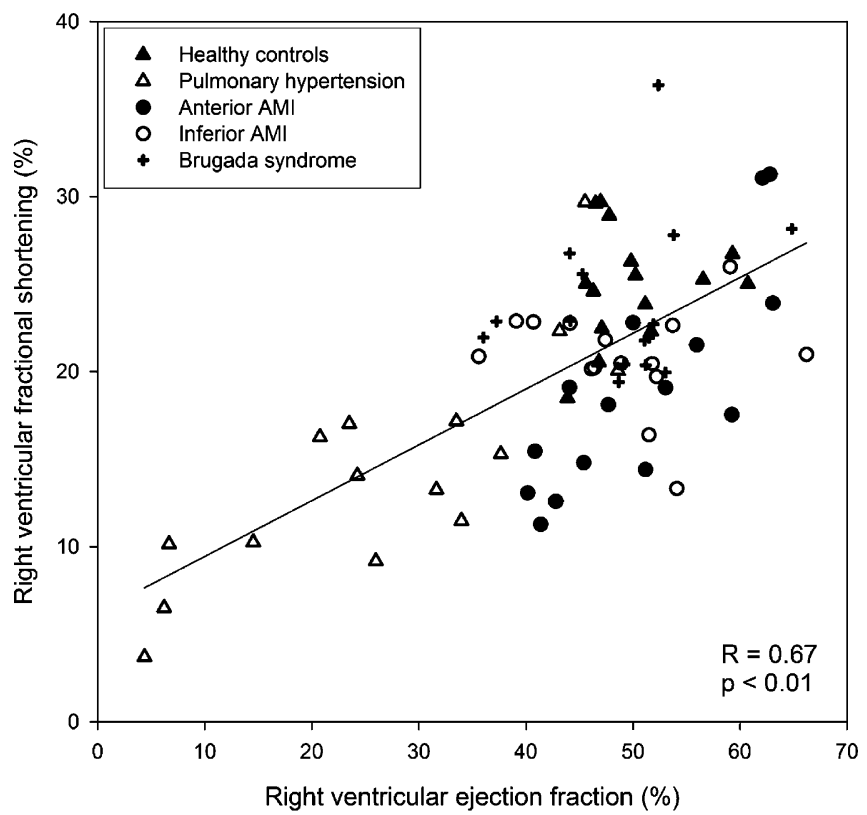

Fig. 2 Linear regression (solid line) of tricuspid annular plane systolic excursion (A) and right ventricular fractional shortening (B) by right ventricular ejection fraction. Symbols represent groups according to legend 
Table 2 The TAPSE and RVFS cut-off values with the highest sensitivity and specificity for predicting RV EF $<35 \%,<40 \%$ and $<45 \%$

\begin{tabular}{|c|c|c|c|c|c|c|}
\hline \multirow[t]{2}{*}{ RV EF } & \multicolumn{3}{|l|}{ TAPSE } & \multicolumn{3}{|l|}{ RVFS } \\
\hline & Cut-off value & Sensitivity & Specificity & Cut-off value & Sensitivity & Specificity \\
\hline$<35 \%$ & 18 & 100 & 73 & 17 & 100 & 86 \\
\hline$<40 \%$ & 17 & 75 & 81 & 17 & 75 & 86 \\
\hline$<45 \%$ & 18 & 70 & 79 & 19 & 67 & 85 \\
\hline
\end{tabular}

The RV EF as estimated by CMR showed significant correlation to TAPSE $(\mathrm{r}=0.62, \mathrm{p}<0.01$, Fig. $2 \mathrm{~A})$. The relationship between RV EF and RVFS was also statistically significant $(r=0.67, p<0.01$, Fig. $2 B)$. There were no significant differences between both correlation coefficients $\left(Z_{o b s}=0.52\right)$. The TAPSE and RVFS cut-off values with the highest sensitivity and specificity for predicting $\mathrm{RV} \mathrm{EF}<35 \%,<40 \%$ and $45 \%$ are listed in Table 2. Figure 3 displays the ROC curves of TAPSE and RVFS to indicate RV EF $<35 \%$. Specificity of RVFS was higher than TAPSE to detect RV dysfunction; however, comparison between the areas under the ROC curves of TAPSE and RVFS revealed no statistical differences.

Figure 4 represents the Bland-Altman analyses of intraobserver and inter-observer variabilities of RV EF and TAPSE measurements. Intra-observer and inter-observer variability of RV EF calculations was low (3\% and 4\%, respectively). Although the intra-observer and interobserver variability of TAPSE measurements were substantially higher ( $7 \%$ and $15 \%$, respectively), the differences in intra- and inter-observer variabilities of both RV EF ( $p=0.74$ and $p=0.40$, respectively) and TAPSE $(p=0.40$ and $p=0.56$, respectively) were not statistically significant.

\section{Discussion}

In the present study, we evaluated the accuracy and performance of a semi-quantitative assessment of RV function in CMR, and the intra-observer and inter-observer reproducibility of this method, compared to the standard quantitative approach. Our data demonstrate that TAPSE in CMR correlates well with 3D volumetric assessment of RV function. Intra-observer reproducibility was better than the inter-observer reproducibility of TAPSE, but inferior to the $3 \mathrm{D}$ volumetric approach, which had comparable intra- and inter-observer variability. Right ventricular fractional shortening, which corrects for RV length, showed also significant correlation with quantitative analysis.

Previous reports have demonstrated a relationship between echocardiographic TAPSE and quantitative assessment of RV function, using different modalities [2224]. In the present study, TAPSE acquired on CMR images showed comparable normal ranges with previous studies in echocardiography $[25,26]$, thus the described method for
CMR seems valid. The reason for a higher level of correlation between TAPSE and RV EF in the present study may be a larger study population with a wider range of RV parameters than previously investigated. In addition, in CMR it is possible to correct TAPSE for RV length, which may offer a reliable semi-quantitative measure in small hearts as well. RVFS tended to correlate better with RV function than TAPSE and indicated impaired RV function with comparable sensitivity but higher specificity than TAPSE. Future research is needed to evaluate its possible statistical superiority and clinical applicability.

In this study we used short axis orientation for volumetric assessment of RV volumes [27-30]. Alfakih and colleagues demonstrated that axial orientation results in better observer variabilities than the short axis orientation [31]. They suggest that the better identification of the pulmonary and tricuspid valves, and the basal slice in the axial orientation are mostly responsible for the differences. In our study, we used the four-chamber view to verify whether the basal short axis slice was part of the RV and should be included in analysis, which resulted in good reproducibility as well. Furthermore, in clinical practice, short axis orientation allows both analysis of left and right ventricular volumes and is therefore most often used.

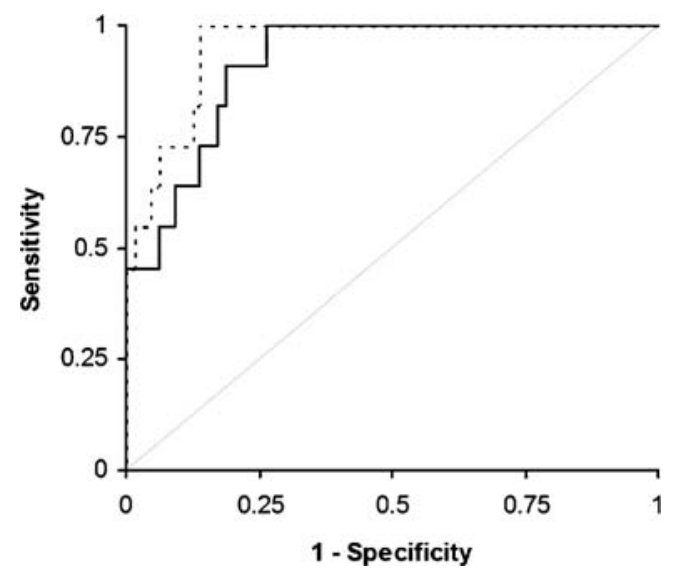

Fig. 3 Receiver-operating characteristic curves of the TAPSE (solid line) and RVFS (dashed line) to indicate right ventricular ejection fraction of less than $35 \%$. Area under the curves was $0.92(0.85-$ $0.99)$ for TAPSE, versus $0.95(0.90-1.00)$ for RVFS. There was no statistical difference between both areas under the curve 
Intra-observer variability of RVEF measurements

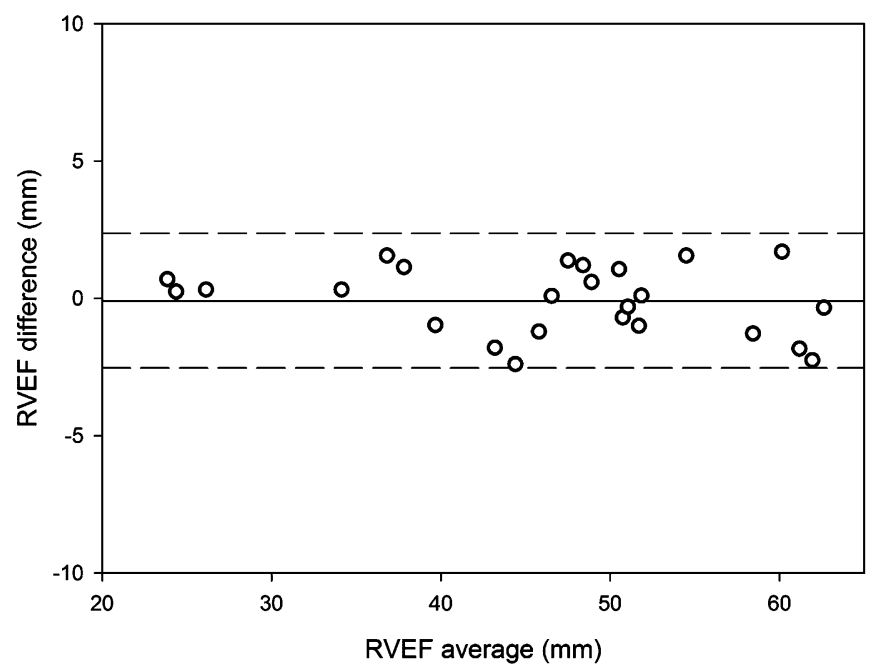

Intra-observer variability of TAPSE measurements

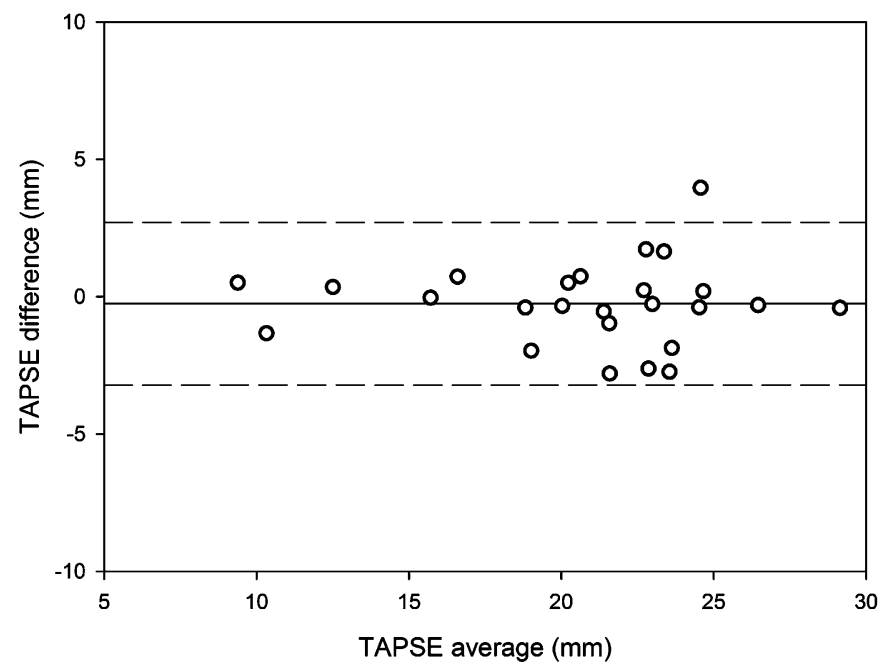

Inter-observer variability of RVEF measurements

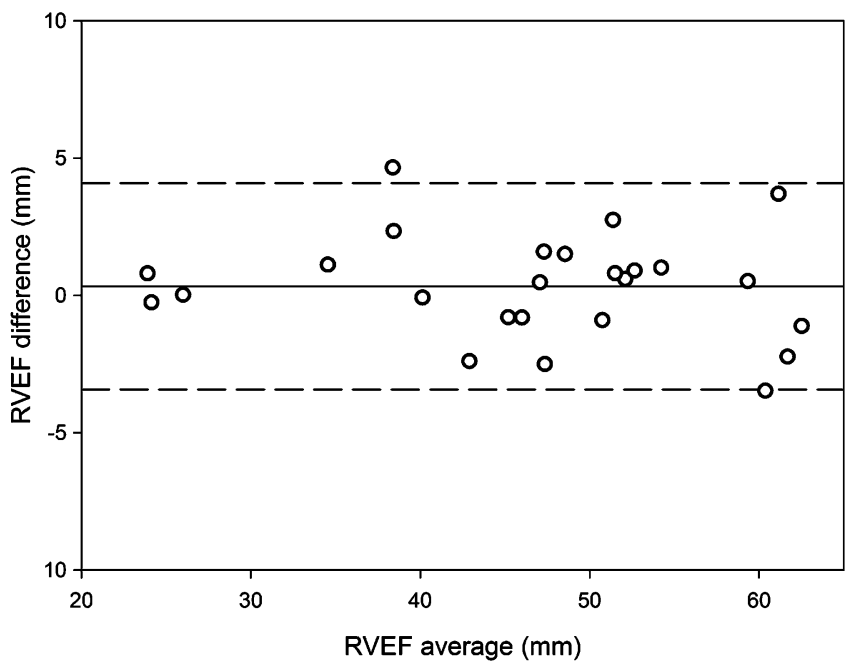

Inter-observer variability of TAPSE measurements

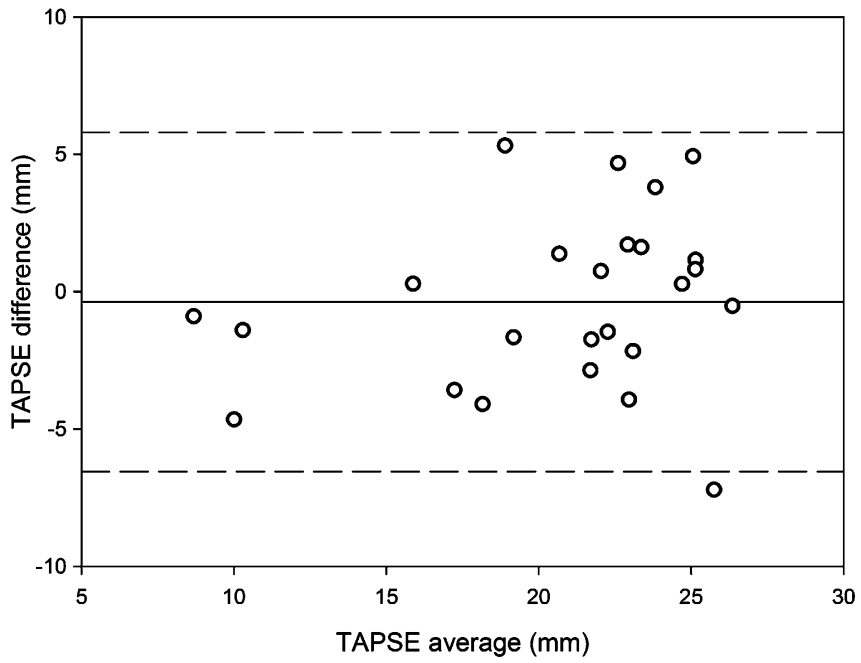

Fig. 4 Bland-Altman plot illustrating the intra- and inter-observer variability of RV EF and TAPSE measurements. Mean difference (solid line) and $95 \%$ limits of agreement (dashed lines) are shown

The status of CMR as a highly reproducible reference standard for the assessment of cardiac function is based on its flexible multi-plane capabilities. The present study offers directions to perform semi-quantitative measurements for right ventricular function using CMR. Since RV function provides important prognostic information, it is desirable to have a screening tool to identify patients with RV dysfunction. Both TAPSE and RVFS were validated for this purpose and showed good performance, but also had important limitations. For routine screening of the RV in a standard clinical CMR examination, TAPSE and RVFS seem reliable and easy methods to identify patients with RV dysfunction. However neither TAPSE nor RVFS were able to distinguish moderate and mildly depressed RV function. For research purposes or when small changes in
RV function may have important clinical consequences, e.g., for the evaluation of response to treatment, the more time-consuming $3 \mathrm{D}$ volumetric approach is preferred to assess RV function.

Acknowledgements The authors would like to acknowledge the careful review of statistical analyses by Dr. Alexander Hirsch. Drs. Robin Nijveldt and Tjeerd Germans were supported by the Netherlands Heart Foundation grants 2003B126 and 2006B213, respectively.

Open Access This article is distributed under the terms of the Creative Commons Attribution Noncommercial License which permits any noncommercial use, distribution, and reproduction in any medium, provided the original author(s) and source are credited. 


\section{References}

1. Bossone E, Duong-Wagner TH, Paciocco G, Oral H, Ricciardi M, Bach DS, Rubenfire M, Armstrong WF (1999) Echocardiographic features of primary pulmonary hypertension. J Am Soc Echocardiogr 12:655-662

2. de Groote P, Millaire A, FoucherHossein C, Nugue O, Marchandise X, Ducloux G, Lablanche JM (1998) Right ventricular ejection fraction is an independent predictor of survival in patients with moderate heart failure. J Am Coll Cardiol 32:948-954

3. Zehender M, Kasper W, Kauder E, Schonthaler M, Geibel A, Olschewski M, Just H (1993) Right ventricular infarction as an independent predictor of prognosis after acute inferior myocardial infarction. N Engl J Med 328:981-988

4. Bleasdale RA, Frenneaux MP (2002) Prognostic importance of right ventricular dysfunction. Heart 88:323-324

5. Grothues F, Moon JC, Bellenger NG, Smith GS, Klein HU, Pennell DJ (2004) Interstudy reproducibility of right ventricular volumes, function, and mass with cardiovascular magnetic resonance. Am Heart J 147:218-223

6. Helbing WA, Bosch HG, Maliepaard C, Rebergen SA, van der Geest RJ, Hansen B, Ottenkamp J, Reiber JH, de Roos A (1995) Comparison of echocardiographic methods with magnetic resonance imaging for assessment of right ventricular function in children. Am J Cardiol 76:589-594

7. Jiang L, Siu SC, Handschumacher MD, Luis GJ, Vazquez de Prada JA, King ME, Picard MH, Weyman AE, Levine RA (1994) Three-dimensional echocardiography. In vivo validation for right ventricular volume and function. Circulation 89:2342-2350

8. Kaul S, Tei C, Hopkins JM, Shah PM (1984) Assessment of right ventricular function using two-dimensional echocardiography. Am Heart J 107:526-531

9. Nichols K, Saouaf R, Ababneh AA, Barst RJ, Rosenbaum MS, Groch MW, Shoyeb AH, Bergmann SR (2002) Validation of SPECT equilibrium radionuclide angiographic right ventricular parameters by cardiac magnetic resonance imaging. J Nucl Cardiol 9:153-160

10. Ueti OM, Camargo EE, Ueti AA, Lima-Filho EC, Nogueira EA (2002) Assessment of right ventricular function with Doppler echocardiographic indices derived from tricuspid annular motion: comparison with radionuclide angiography. Heart 88:244-248
11. Jauhiainen T, Jarvinen VM, Hekali PE, Poutanen VP, Penttila A, Kupari M (1998) MR gradient echo volumetric analysis of human cardiac casts: focus on the right ventricle. J Comput Assist Tomogr 22:899-903

12. Pennell DJ, Sechtem UP, Higgins CB, Manning WJ, Pohost GM, Rademakers FE, van Rossum AC, Shaw LJ, Yucel EK (2004) Clinical indications for cardiovascular magnetic resonance (CMR): Consensus Panel report. Eur Heart J 25:1940-1965

13. Beygui F, Furber A, Delepine S, Helft G, Metzger JP, Geslin P, Le Jeune JJ (2004) Routine breath-hold gradient echo MRI-derived right ventricular mass, volumes and function: accuracy, reproducibility and coherence study. Int J Cardiovasc Imaging 20:509-516

14. Grothues F, Moon JC, Bellenger NG, Smith GS, Klein HU, Pennell DJ (2004) Interstudy reproducibility of right ventricular volumes, function, and mass with cardiovascular magnetic resonance. Am Heart J 147:218-223

15. Kaul S, Tei C, Hopkins JM, Shah PM (1984) Assessment of right ventricular function using two-dimensional echocardiography. Am Heart J 107:526-531

16. Ueti OM, Camargo EE, Ueti AA, Lima-Filho EC, Nogueira EA (2002) Assessment of right ventricular function with Doppler echocardiographic indices derived from tricuspid annular motion: comparison with radionuclide angiography. Heart 88:244-248

17. Hammarstrom E, Wranne B, Pinto FJ, Puryear J, Popp RL (1991) Tricuspid annular motion. J Am Soc Echocardiogr 4:131-139

18. Sievers B, Brandts B, Franken U, Trappe HJ (2004) Single and biplane TrueFISP cardiovascular magnetic resonance for rapid evaluation of left ventricular volumes and ejection fraction. J Cardiovasc Magn Reson 6:593600

19. Wilde AA, Antzelevitch C, Borggrefe M, Brugada J, Brugada R, Brugada P, Corrado D, Hauer RN, Kass RS, Nademanee K, Priori SG, Towbin JA (2002) Proposed diagnostic criteria for the Brugada syndrome. Eur Heart J 23:1648-1654

20. Hanley JA, McNeil BJ (1983) A method of comparing the areas under receiver operating characteristic curves derived from the same cases. Radiology 148:839-843

21. Bland JM, Altman DG (1986) Statistical methods for assessing agreement between two methods of clinical measurement. Lancet 1:307-310
22. Kaul S, Tei C, Hopkins JM, Shah PM (1984) Assessment of right ventricular function using two-dimensional echocardiography. Am Heart J 107:526-531

23. Ueti OM, Camargo EE, Ueti AA, Lima-Filho EC, Nogueira EA (2002) Assessment of right ventricular function with Doppler echocardiographic indices derived from tricuspid annular motion: comparison with radionuclide angiography. Heart 88:244-248

24. Kjaergaard J, Petersen CL, Kjaer A, Schaadt BK, Oh JK, Hassager C (2006) Evaluation of right ventricular volume and function by 2D and 3D echocardiography compared to MRI. Eur J Echocardiogr 7:430-438

25. Kjaergaard J, Petersen CL, Kjaer A, Schaadt BK, Oh JK, Hassager C (2006) Evaluation of right ventricular volume and function by 2D and 3D echocardiography compared to MRI. Eur J Echocardiogr 7:430-438

26. Tamborini G, Pepi M, Galli CA, Maltagliati A, Celeste F, Muratori M, Rezvanieh S, Veglia F (2006) Feasibility and accuracy of a routine echocardiographic assessment of right ventricular function. Int J Cardiol:

27. Grothues F, Moon JC, Bellenger NG, Smith GS, Klein HU, Pennell DJ (2004) Interstudy reproducibility of right ventricular volumes, function, and mass with cardiovascular magnetic resonance. Am Heart J 147:218-223

28. Kjaergaard J, Petersen CL, Kjaer A, Schaadt BK, Oh JK, Hassager C (2006) Evaluation of right ventricular volume and function by 2D and 3D echocardiography compared to MRI. Eur J Echocardiogr 7:430-438

29. Maceira AM, Prasad SK, Khan M, Pennell DJ (2006) Reference right ventricular systolic and diastolic function normalized to age, gender and body surface area from steady-state free precession cardiovascular magnetic resonance. Eur Heart J 27:2879-2888

30. Tandri H, Daya SK, Nasir K, Bomma C, Lima JA, Calkins H, Bluemke DA (2006) Normal reference values for the adult right ventricle by magnetic resonance imaging. Am J Cardiol 98:16601664

31. Alfakih K, Plein S, Bloomer T, Jones T, Ridgway J, Sivananthan M (2003) Comparison of right ventricular volume measurements between axial and short axis orientation using steady-state free precession magnetic resonance imaging. J Magn Reson Imaging 18:25-32 